\title{
ORAZIO, O DELL'ARTE DI MANGIAR BENE
}

\author{
PAOLO FEDELI $\left(^{*}\right)$
}

SunTO. - Nelle Satire di Orazio si trova la testimonianza di diverse tipologie di pasto dei Romani : il poeta ci descrive sia il pasto frugale (Ofello: Sat. II 2), sia le raffinatezze gastronomiche (Cazio: Sat. II 4), sia il banchetto fastoso (Nasidieno: Sat. II 8). Quanto a Orazio, egli non nasconde la propria preferenza per l'alimentazione semplice e il suo rifiuto dei piatti troppo elaborati.

$* * *$

RESUMÉE. - Dans les Satires d'Horace on trouve le témoignage des différentes typologies du repas des Romains: le poète nous décrit soit le repas frugal (Ofellus: Sat. II 2) soit les raffinements gastronomiques (Catius: Sat. II 4) soit le banquet grandiose (Nasidiénus: Sat. II 8). Quant à Horace, il ne dissimule pas sa préférence pour la nourriture simple et son refus des mets trop soignés.

1. «Chi mangia bene - ce lo garantisce Orazio nella VI epistola del I libro - vive felice». Chi, allora, meglio di Orazio potrà guidarci in un ideale percorso gastronomico, Orazio che nei suoi sermones (le Satire e le Epistole) ha concesso ampio spazio ai personaggi e alle situazioni della vita di tutti i giorni negli ultimi decenni del I sec. a.C.? Nel trattare di gastronomia in versi, egli s'inserisce in un'antica tradizione, che a Roma aveva avuto un illustre antecedente addirittura negli Hedyphagetica del pater Ennius: da lui, ovviamente, non dovremo attenderci la dettagliata presentazione di ricette culinarie, ma la testimonianza del gusto dei Romani del tempo suo e del loro rapporto con l'alimentazione. La testimonianza di Orazio è preziosa perché ci documenta le diverse tipologie dei pasti dei Romani: dal pasto frugale (Ofello nella

(*) Università degli Studi di Bari, Italia. E-mail: p.fedeli@ria.uniba.it 
Sat. II 2) alla raffinata gastronomia (Cazio nella Sat. II 4) al banchetto grandioso, di tipo trimalchionico (Nasidieno nella Sat. II 8).

La giornata del cittadino romano prevedeva, sostanzialmente come la nostra ma con modalità diverse, tre momenti di contatto col cibo: frugale era la prima colazione (ientaculum), di solito con pane e formaggio, frutta, miele e un'abbondante bevuta di acqua fresca. È ovvio che questa sia la prima colazione 'standard', ma i cibi variavano, e non solo di quantità, a seconda dei ceti sociali e dei mestieri: Catone, nel suo De agricultura, ci attesta che per i contadini, destinati al duro lavoro dei campi durante l'intera giornata, le razioni di cibo erano per forza di cose ben più abbondanti e sostanziose. Rapido e poco impegnativo era il prandium, uno spuntino a base di pane e salumi, uova e frutta, molto spesso gli avanzi del giorno prima; si trattava di un boccone freddo da ingerire in poco tempo e di solito in piedi.

Il pasto principale era la cena, particolarmente impegnativa quando c'erano invitati di riguardo. In tali circostanze la cena aveva inizio nel pomeriggio e durava sino a notte fonda, contraddistinta da tre momenti (gustatio, primae mensae, secundae mensae). La gustatio - il nostro antipasto - era abbondante: immancabili le uova, le olive (specie quelle nere), le verdure, i funghi, i formaggi, il tutto accompagnato da vino mielato (il mulsum). Seguivano le primae mensae, con carne, prevalentemente di maiale, e pesce condito con salse abbondanti e ben diverse dalle nostre (i pesci, ad esempio, potevano essere conditi con salse di susine, di albicocche, di mele cotogne, la cacciagione con miele, vino, olio, menta, aceto, ma anche con pepe e con condimenti forti); nelle primae mensae non si beveva più il mulsum, ma vino allungato. La cena era chiusa dalle secundae mensae (il nostro 'dessert'), in cui oltre ai dolci al miele (abituale dolcificante anche in pasticceria), a fare la parte del leone era la frutta: immancabili le mele, alla fine. Se si considera che la cena aveva inizio con le uova nella gustatio e si concludeva con le mele nelle secundae mensae, ben si capisce come l'oraziano ab ovo usque ad mala (Sat. I 3.6-7) sia un modo perfetto di designare il pasto completo.

Al termine delle secundae mensae aveva inizio la parte 'ludica' della cena, nella quale si continuava a bere vino durante gli ameni conversari. Ovviamente quantità e varietà dei cibi erano in stretto rapporto con le possibilità economiche e con la presenza di invitati di riguardo; negli antipasti i ricconi potevano permettersi le ostriche del Circeo, i ricci di mare, i crostacei; invece le cene dei plebei erano di solito a base di farro, di minestre o di polente, di verdure, di olive e di frutta; sulla 
loro tavola la carne faceva la sua comparsa solo in circostanze eccezionali, per condire il pesce ci si doveva accontentare della salamoia e i farinacei la facevano da padroni: d'altronde è Orazio stesso a confessare che, quando i morsi della fame si fanno sentire, un tozzo di pane con un po' di sale serve egregiamente a calmare stomachum latrantem (Sat. II 2.17-18).

2. Quella di Ofello, nella II satira del II libro, non è una sistematica descrizione di una pur modesta cena, ma una serie di consigli per la scelta dei cibi più sani. Ofello è un contadino di Venosa dotato di una rozza ma efficace saggezza, che tesse l'elogio della vita frugale della campagna e l'oppone alle raffinatezze della vita in città: in campagna non hanno importanza né il lusso né gli artifici, mentre in città solo quando si è sfiniti per la caccia o per gli esercizi fisici non si rifiutano i cibi frugali.

Che quella di Ofello intenda essere una cucina frugale lo si comprende già dalla sua definizione della fame come il migliore dei condimenti, che riprende un motivo topico nella diatriba dei Cinici: prodigo di precetti di saggezza popolare, egli muove dal principio che i cibi più appariscenti e costosi non sono necessariamente più saporiti di quelli umili e poco appariscenti. Non è detto, ad esempio, che un prezioso pavone, dall'elegante coda variopinta, a tavola debba esser preferito a una modesta gallina, di sicuro meno bella a vedersi ma certamente più saporita; e poi, le piume del pavone non sono commestibili. Eppure i ricconi erano capaci di sperperare una fortuna pur di servire in tavola carne di pavone (II 2.23-28). Si capisce bene che quello di Ofello vuole essere l'elogio del principio del giusto mezzo, al riparo dagli eccessi nell'un senso e nell'altro (II 2.63-69): la frugalità non ha nulla a che vedere con la sordidezza di un tal Avidieno, detto il Cane perché faceva una vita da cani, cibandosi di olive rancide e di cornioli e bevendo un vino ormai andato all'aceto. Nei giorni di festa Avidieno condiva i cavoli con due misere gocce d'olio, ma in compenso abbondava in aceto (II 2.53-62).

Ofello tesse l'elogio del cibo frugale perché è convinto dei suoi benefici per la salute e condanna la varietà, che è nociva e produce disastri intestinali: il cibo semplice, egli dice, scende nello stomaco senza alcuna difficoltà; invece un miscuglio di cibi raffinati - dal lesso all'arrosto, dalle ostriche ai tordi - è capace di produrre una vera e propria rivoluzione (II 2.70-76). E poi, chi pratica un morigerato regime alimentare può permettersi qualche licenza, mentre se lo facesse un cra- 
pulone, rovinato dal cibo ingurgitato nel corso degli anni, rischierebbe di tirare le cuoia (II 2.70-93). Chi è dedito alla crapula, dice Ofello, lo si riconosce subito dal volto flaccido e pallido: chi si trova in condizioni fisiche talmente penose deve astenersi dal mangiare cibi raffinati, come le ostriche, gli scari dei lontani mari orientali, le rare ed esotiche pernici bianche (II 2.16-22). Non sarebbe in grado, d'altronde, neppure di gustarne gli invitanti sapori e anche i cibi freschissimi gli sembrerebbero puzzolenti.

Fin qui non c'è molto di nuovo perché, sia pur associati a personali divagazioni, quelli di Ofello sono principi ampiamente accolti dall'etica epicurea, secondo la quale i cibi frugali preservano la salute e difendono l'uomo dai colpi della fortuna. I pur nobili principi filosofici, però, servono poco nelle situazioni di emergenza: che fare, ad esempio, se un ospite affamato si presenta in casa all'improvviso? Nella dispensa, ammonisce Ofello, non deve mai mancare la carne di cinghiale: non quella fresca, però, ma quella vecchia, frollata, che sarà pure rancida $\mathrm{e}$ maleodorante, ma dura a lungo, è commestibile, e ti salva nelle situazioni critiche (II 2.89-93). Può capitare, poi, che il dominus giunga nella propria villa sulle rive del mare e non trovi lo schiavo addetto alla custodia delle provviste (il promus); per colmo di sfortuna il mare è in tempesta e impedisce la pesca: in una circostanza a tal punto disgraziata l'etica della frugalità dice che un po' di pane e sale è sufficiente a placare la fame; d'altronde il gusto - ammonisce Ofello - non risiede nel profumo di costose vivande, ma nel proprio appetito (II 2.16-22). Ofello non rifugge dalla vivace polemica: se la prende con gli pseudointenditori, quelli che si ritengono in grado di capire se un pesce sia stato pescato nei pressi della foce del Tevere o in alto mare, oppure se sia stato trascinato dalla corrente fra i due ponti dell'isola Tiberina, dove sboccava la cloaca Massima fornendo ai pesci abbondante nutrimento (II 2.29-32). Se la prende anche con quanti si vantano d'aver inserito nelle primae mensae triglie dal peso eccezionale di tre libbre, fatte a pezzi per la loro eccessiva grandezza (II 2.33-35).

Il pregio delle parole di Ofello risiede nella possibilità che esse ci offrono di cogliere fasi di mutamento nel codice alimentare romano, che secondo lui si era 'corrotto' dopo la conclusione delle guerre con Cartagine e sempre più era attratto da costosissimi cibi esotici (II 2.4648): sappiamo bene, d'altronde, che nelle varie leges sumptuariae ci si preoccupò costantemente di mettere un freno al dilagare del lusso in campo alimentare, ma sappiamo anche che le disposizioni di legge veni- 
vano regolarmente eluse. Eppure, sostiene Ofello (II 2.44-52), anche nelle mense dei ricchi si assiste a una rivalutazione della cucina povera, come nel caso delle uova e delle olive; d'altronde i gusti dei ghiottoni mutano col passare del tempo, e mentre in passato nessuno osava cibarsi di rombi e cicogne, ora a Roma i giovani li prediligono (II 2.44-52).

In quanto a lui, è costretto a un'alimentazione frugale, oltreché da una scelta vagamente filosofica, dalla modesta condizione di vita: di recente è divenuto fittavolo nel campicello che un tempo era suo e che ora è stato diviso (II 2.114-115). Nei giorni lavorativi il suo cibo abituale è costituito da legumi accompagnati da zampetto di prosciutto affumicato (II 2.117); rari sono per lui i banchetti e legati alla visita di un ospite o di un vicino, e in essi il cibo ripropone a livello alimentare la topica opposizione fra città e campagna: i pesci comprati nei mercati cittadini cedono il posto, nelle primae mensae di Ofello, a un pollo o a un capretto, mentre le secundae mensae propongono come 'dessert' noci e fichi secchi divisi in due (II 2.118-122). Con questa atmosfera di sana semplicità campestre s'accorda, al termina delle secundae mensae, la fase ludica della cena: banditi i dotti conversari, il divertimento consiste in allegre bevute di vino, senza sottostare alle leggi del magister convivii.

3. Dalla cucina povera di Ofello passiamo a quella che ha pretese di raffinatezza, perché dispensatore di raffinati precetti gastronomici pretende di essere il protagonista della IV satira del II libro: Cazio, che il poeta trova immerso in profondi pensieri, sta cercando di mandare a mente con l'aiuto della mnemotecnica una serie d'importanti concetti. Si viene subito a sapere, però, che si tratta di precetti gastronomici: sicché quella da lui impartita ad Orazio è una vera e propria ars ... cenandi, nella quale - con alcune inevitabili divagazioni - viene rispettato lo svolgimento di una cena, che pretende di segnalarsi per la sua perfetta ricercatezza. In questo caso, dunque, abbiamo la possibilità di seguire nella loro successione le tre fasi della cena romana (gustatio, primae mensae, secundae mensae). Gli antipasti (II 4.12-34 gustatio) prevedono uova, ortaggi, funghi, ostriche, ricci di mare, molluschi, il tutto accompagnato da abbondanti bevute di mulsum. Qua e là Cazio dispensa saggi precetti: mentre ai giorni nostri si preferiscono le uova rotondeggianti, egli predilige quelle dalla forma allungata, a suo dire più saporite perché migliore è il loro albume e perché esse contengono un tuorlo maschio (era questo un pregiudizio ampiamente diffuso nel mondo antico). Fra gli ortaggi il preferito, manco a dirlo, è il cavolo, sin dai 
tempi di Catone considerato rimedio sicuro per tutti i mali: veniamo a sapere, però che si considerava più saporito quello che cresceva su terreni secchi, mentre era quasi sempre insipido quello abbondantemente innaffiato negli orti. Nella gustatio i funghi costituiscono una novità, visto che altrove non c'è testimonianza della loro presenza in questa parte della cena: era probabilmente la loro pericolosità, associata all'incapacità di saper sempre distinguere le specie commestibili dalle letali, a suscitare diffidenza; tuttavia essi non mancavano sulla tavola dei buongustai, ma come ricercata raffinatezza piuttosto che come sistematica presenza: d'altronde, si chiederà un secolo dopo Plinio il Vecchio (Hist. XXII 47, 97), quae voluptas tam ancipitis cibi? Per parte sua Cazio sviluppa un confronto tra funghi prataioli e funghi di bosco, a tutto vantaggio dei primi, con i quali non si rischia.

I molluschi pregiati vengono elencati con competenza, nella certezza che sia il periodo della luna nuova a rendere più piene le ostriche: oltre al periodo, anche il luogo favorisce la crescita di ostriche e conchiglie: la vongola verace del lago Lucrino è migliore del murice che si trova fra le rocce del golfo di Baia, le ostriche più ricercate sono quelle del Circeo e i ricci di mare (echini) più pregiati sono quelli di capo Miseno. La sezione dedicata ai molluschi indica in Taranto (II 4.34) la città che a ragione può vantarsi di produrre i più gustosi pettini di mare, cioè quelle conchiglie oggi comunemente chiamate 'cappe sante'. Quanto al mulso, lo sciroppo di miele misto a vino Falerno che accompagna gli antipasti, è bene che non contenga un Falerno troppo robusto, perché se così fosse rischierebbe di danneggiare la circolazione sanguigna.

I cibi delle primae mensae, dai pesci alla selvaggina (II 4.35-46), a detta di Cazio devono sottostare a un nobile precetto sulla necessità di conoscer bene la sottile filosofia dei sapori (II 4.36). L'insegnamento che da questa massima scaturisce viene applicato subito al mondo ittico (II 4.37-39): non basta spazzolare il bancone del pescivendolo, se si ignora a quali pesci si addica meglio la salsa e quali, invece, arrostiti abbiano l'effetto di far sdraiare di nuovo sul gomito il convitato già sazio (c'è da tener presente la consuetudine dei Romani di mangiare sdraiati, poggiandosi sul gomito sinistro; chi si sentiva sazio finiva per sdraiarsi esausto sui cuscini, e per fargli riassumere la primitiva posizione sul gomito bisognava ridestargli l'appetito). La bontà del pesce arrosto, a detta di Cazio, dipende dalla qualità e dalla quantità dell'olio con cui lo si condisce. Dai pesci si passa alla selvaggina: da sempre il cinghiale costituiva la portata principale dei banchetti, e ben si giustifica 
che Cazio lo esiga di elevata qualità: egli mette a confronto il cinghiale di montagna (l'Umber aper) e quello di pianura (il Laurens aper), e sentenzia che la superiorità del primo è garantita sia dall'alimentazione con le ghiande di leccio (II 4.40) che ne rendono compatta e saporita la carne, sia dal peso notevole. Insieme ai cinghiali i Romani erano soliti allevare le lepri, i cervi, i caprioli: Cazio sconsiglia la carne dei caprioli allevati nei terreni a vigneto (II 4.43), che non giudica sempre commestibile, perché il cibarsi di pampini non conferiva alla carne di capriolo un sapore gradevole. In quanto, poi, alla lepre, gli intenditori preferivano sistematicamente la spalla di quella che aveva già figliato (II 4.44).

Avviandosi al termine del suo sproloquio, Cazio si decide a svelare la sorpresa finora tenuta in serbo, perché si tratta di una sua specialità: la introduce addirittura (II 4.63) ricorrendo a un altisonante esordio enniano (est operae pretium), che lascia presagire un argomento di straordinaria importanza, mentre il verbo pernoscere fa capire, grazie al suo prefisso, che un tale argomento verrà scrutato sino in fondo, in tutte le sue pieghe: per di più si tratta di pernoscere una duplicis iuris natura. A sorpresa si viene a sapere che non si tratta di conoscere a fondo la rerum natura di lucreziana memoria, bensì - nell'economia della cena - la composizione della salsa doppia. Orazio gioca sul doppio senso, giuridico e culinario di ius ("legge", ma anche "sugo"). Dato che l'indagine deve essere seria e approfondita perché deve condurre alla conquista della verità, Cazio illustra dapprima la composizione della salsa semplice (olio dolce mescolato con vino da taglio e salamoia). La salamoia, che si ottiene con le viscere di tonno mescolate a quelle di altri pesci, non dev'essere di tipo comune, ma deve venire da Bisanzio: per rendere più incisive le sue parole, Cazio parla di salamoia che fa 'puzzare' il barile di Bisanzio. Ed ecco il segreto della salsa doppia (II 4.67-68): non appena la mistura sarà giunta ad ebollizione con l'aggiunta di erbe aromatiche tritate e si sarà riposata dopo che vi sarà stato versato zafferano di Corico, bisognerà aggiungere olio di Venafro.

Dalle salse si passa alla frutta, a cominciare dalle mele, che vengono servite all'inizio delle secundae mensae. Come prima era avvenuto per il cinghiale umbro e per quello di Laurento, ora vengono messe a confronto le mele picene e quelle di Tivoli, in una competizione che vede le prime avere la meglio per il sapore, le seconde per l'aspetto. In quanto all'uva, la cosiddetta vennuncula (o venucula) va conservata in grandi vasi di terracotta (II 4.71), quella di Albano va fatta stagionare essiccandola al fumo. L'uva di Albano consente a Cazio di autoelogiarsi per i suoi 
meriti d'inventor (II 4.73-75): egli rivendica di essere stato il primo a servirla a tavola insieme alle mele, il primo a servire la feccia del vino mescolata con allec (cioè quanto, nella preparazione del garum, rimaneva delle interiora dei pesci macerati in salamoia), il primo a offrire ai commensali pepe bianco (cioè la qualità di pepe meno forte) passato al setaccio, insieme a sale nero (forse perché mescolato con altre spezie).

Scarna e convenzionale, a confronto con tanta ricercatezza, è la lista dei vini (il Massico, il Sorrentino, l'immancabile Falerno): il Massico, dall'omonimo monte fra Lazio e Campania, dovrà restare esposto a cielo sereno, in modo che le sue impurità siano attenuate dall'aria notturna e svanisca quell'aroma intenso che fa ubriacare; lo si può filtrare, ma col risultato di fargli perdere il sapore genuino. Quanto al Falerno, la sua feccia mescolata al Sorrentino gli conferisce una maggiore robustezza: chi lo fa dà prova di grande accortezza, specie se poi fa precipitare sul fondo del recipiente la feccia con uova di piccione, il cui tuorlo scendendo trascina con sé tutte le impurità (II 4.51-62).

4. Nella cena di Nasidieno di Sat. II 8 si può facilmente scorgere un'anticipazione del trimalchionico banchetto petroniano nella ricerca dello stupefacente, anche se il protagonista dell'ultima satira oraziana si limita alle stravaganti portate. Il disordine nella presentazione della cena, col mancato rispetto della canonica successione delle sue fasi ben distinte, fa parte della ricerca di una fastosa originalità che caratterizza il padrone di casa: è contrario alla norma il fatto stesso che il convito abbia inizio addirittura a mezzogiorno, per di più non con la gustatio ma con la comissatio, con un totale sconvolgimento della scansione reale della cena. Nella comissatio Nasidieno non si accontenta del Cecubo, pregiatissimo vino del Lazio meridionale, ma ai suoi ospiti offre anche vino di Chio, puro tuttavia e non mescolato con acqua di mare com'era consuetudine con i vini provenienti dalla Grecia: però egli mette in chiaro che se qualcuno preferisce i pregiatissimi Albano e Falerno, entrambi sono disponibili in cantina. Emblematica è anche la gustatio: negli antipasti si cercava di non esagerare con i cibi pesanti, e invece Nasidieno fa servire a tavola come antipasto un cinghiale intero, per di più in salsa abbondante, tanto per stuzzicare l'appetito; si tratta, a scanso di equivoci, di un cinghiale lucano, cacciato quando spirava uno scirocco leggero (se, infatti, lo scirocco fosse stato opprimente, la carne del cinghiale sarebbe frollata rapidamente). Nell'enorme vassoio fa corona al cinghiale un ricco contorno di ravanelli piccanti, lattuga, 
radicchio, carote, con feccia di vino di Cos e l'immancabile salsa di pesce.

Dopo un simile esordio non stupisce che le portate esibiscano una preparazione diversa da quella abituale e si segnalino tutte per la loro originalità. È questo il frutto di un'accorta opera di mimesi da parte del cuoco, grazie alla cui maestria i convitati - proprio come avverrà nella cena petroniana - credono di mangiare una vivanda, ma si accorgono solo in un secondo momento che si tratta di ben altro: ne abbiamo la prova allorché Nasidieno offre a Fundanio (che ad Orazio racconta lo svolgimento della cena) interiora di pesce passero e di rombo di un sapore mai gustato prima e gli svela, poi, di che si tratti. Incoraggiato dalla meraviglia suscitata dal sapore nuovo dei filetti di rombo, Nasidieno istruisce Fundanio sulle virtù dei melimela, cioè delle mele dolcissime come il miele, che sono di un colore rosso vivo se vengono colte nel periodo della luna calante.

A questo punto viene servita a tavola una murena con contorno di gamberetti che sguazzano nella salsa: prima che gli invitati si lancino su un cibo così prelibato, Nasidieno interviene col solito tono didascalico per spiegare che, come nel caso del cinghiale lucano, l'eccellenza della murena dipende dal momento in cui la si è catturata, che è poi quello della sua gravidanza. Straordinario è anche il sugo, perché l'olio proviene dalla zona del Venafro ed è di prima spremitura, il garum non è di produzione locale ma giunge dalla penisola iberica, il vino oltre ad essere adeguatamente invecchiato non è greco, ma italico: per la buona riuscita della salsa, però, bisogna versarlo durante la cottura ed è necessario aggiungere pepe bianco e un po' d'aceto, beninteso quello ottenuto facendo fermentare il vino greco di Metimna (II 8.42-50). Le salse costituiscono la specialità di Nasidieno, che orgogliosamente rivendica il merito di aver insegnato a cuocere nel sugo ruchette verdi ed enule amare, anche se riconosce cavallerescamente che non spetta a lui, ma a un tal Curtillo, la priorità nell'aver inserito nel sugo anche i ricci di mare: non lavati, però, perché quello che i frutti di mare secernono, nell'espellere l'acqua marina assorbita, è più saporito della salamoia.

All'improvviso un baldacchino appeso al soffitto precipita rovinosamente sul piatto con la murena e i gamberetti in salsa abbondante: si sfiora la catastrofe, ma una volta ristabilita la calma viene portata a tavola, su un enorme vassoio, una gru già divisa in porzioni dallo scissor e abbondantemente cosparsa di sale e di farro, con fegato d'oca, fichi maturi e spalle di lepre staccate dai lombi, perché giudicate molto più 
gustose. Servire una gru a tavola non doveva essere abituale, se non altro per problemi di gusto: a detta di Plinio il Vecchio, infatti (Hist. X 30.60), già Cornelio Nepote sosteneva che alle gru sono di gran lunga preferibili le cicogne; tuttavia la raffinatezza di Nasidieno consiste anche nel saper valorizzare cibi di gusto non comune, anticipando talora mode future. Ai convitati vengono serviti, infine, petti di merlo arrosto e colombacci privi di cosce: anche in questo caso, come in quello della lepre, la peculiarità consiste nel rifiuto dei quarti posteriori, che invece erano apprezzati da molti. Come al solito Nasidieno comincia a descrivere le caratteristiche delle prelibate vivande; ma i convitati non ne possono più e si vendicano decidendo di non toccare più cibo (II 8.80-95).

5. Ofello, Cazio, Nasidieno rappresentano tre modi diversi di concepire il rapporto dei Romani col cibo: ma, si dirà, in quale dei tre si riconosce Orazio? Se si deve dar credito ai suoi versi, non c'è dubbio che in campo alimentare le sue simpatie vadano ad Ofello: d'altronde il suo è l'atteggiamento che ci si attende da chi del modus ha fatto sempre il suo ideale di vita. Se, dunque, la predilezione per il cibo frugale non dipende da taccagneria, è in esso che Orazio vede realizzato il giusto mezzo in campo alimentare. Gli accenni ai suoi pasti hanno come sfondo, quasi esclusivamente, l'ambiente campestre, e le sue cene frugali preludono a un placido riposo nei pressi di un ruscello (Epist. I 14.3: cena brevis iwvat et prope rivum somnus in unda). Quando, invece, si trova a Roma, Orazio sospira al pensiero della campagna lontana: lì può dimenticare la vita caotica dell'Urbe, lì i cibi saranno pure frugali ma hanno il vantaggio di essere genuini e le cene vedono raccolti attorno al focolare il padrone, gli amici, gli schiavi. Ognuno beve quanto vuole, libero dalle rigide leggi del convito, e i conversari sono alla buona: bandite le dotte disquisizioni letterarie e filosofiche, si raccontano favolette che abbiano una loro morale: come quella del topo di città e del topo di campagna, che vuol dimostrare come la campagna non si limiti a offrire una vita al riparo dai pericoli, ma consenta di nutrirsi di cibi semplici, ben diversamente dalla città, che espone a rischi continui nonostante gli splendori della mensa (Sat. II 6.59-117). O rus, quando ego te adspiciam? «O campagna - egli sospira - quando ti rivedrò? (...) Quando mi verranno servite a tavola le fave, parenti di Pitagora, e verdure ben condite di grasso lardo?» (II 6.60; ivi, 63-64). Orazio, dunque, ricorda con nostalgia i prodotti dei campi, in primo luogo le fave, con 
una spiritosa allusione al divieto pitagorico di mangiarle, poi le verdure, purché siano ben condite. Il suo è l'elogio di una cucina sostanzialmente vegetariana, in cui tuttavia non deve mancare un po' di carne di maiale. Dato che il vino scioglie la lingua e favorisce la conversazione, al termine della cena ognuno ha il diritto di bere quanto vuole e non deve sottostare alle leggi che regolano il simposio cittadino.

La preferenza di Orazio per il cibo semplice non cambia neppure quando egli descrive le sue giornate di civis a Roma (Sat. I 6.111-115, nella traduzione di Carlo Carena):

vado soletto dovunque mi piace,

chiedo il prezzo del farro e dei legumi,

percorro il circo ingannatore e spesso

il foro a sera; mi fermo, riguardo

gli indovini, e alla fine torno a casa

a un piatto di frittelle, porri e ceci.

Farro e legumi non mancano mai, ma a incuriosire è quello che Carena traduce come "un piatto di frittelle, porri e ceci". Me ad porri et ciceris refero laganique catinum, recita il testo latino, ma in merito al significato di laganum non v'è certezza, tanto che c'è chi pensa a una specie di pasta al forno vegetariana e chi, invece, a un minestrone a base di porri e di ceci. Le altre attestazioni del termine, in Isidoro (Orig. XX 2.17) e nel commento ad loc. dello Pseudoacrone, fanno pensare a sottili sfoglie di farina, fritte nell'olio e successivamente cosparse di una salsa a base di miele, pepe e probabilmente garum e farcite di verdure e legumi: un piatto semplice, dunque, in puro stile vegetariano, in un ambiente modesto (un disadorno piano di marmo bianco, un mestolo, una saliera e un'ampolla). Quando, anni dopo, nella prima del II libro delle epistole, nella raffigurazione del poeta che in un mondo amante del lusso si distingue per la sua vita semplice, Orazio disegnerà il proprio autoritratto, in un rapido accenno alle abitudini alimentari metterà in chiaro che a lui basta cibarsi di legumi, anzi addirittura della buccia dei legumi, e di pane di seconda scelta: l'epistola, com'è noto, è diretta ad Augusto e non sarà un caso che Svetonio (Aug. 76.1) attribuisca ad Augusto gli stessi gusti del poeta per i cibi frugali e per il pane di seconda scelta (cibi ...minimi erat atque vulgaris fere: secundarium panem ... appetebat). Identica è l'atmosfera di semplicità in cui si colloca l'invito a cena rivolto a Torquato nell'epistola I 5: certo, il tema dell'invito a una cena modesta appartiene a una tradizione letteraria antica almeno quanto Bacchilide: 
è indubbio, però, che al regime di vita di Orazio e al suo ideale del modus ben si addice il culto della mediocritas, che nell'invito a Torquato si manifesta nell'esigenza di adattarsi a divani angusti, a un vasellame rustico, a un cibo modesto e a un vino di non eccelsa qualità.

Questa scelta di vita improntata alla morigeratezza ci appare saggia e fonte di benefici per la salute. Non so, tuttavia, se la penserete allo stesso modo, una volta ascoltati alcuni consigli di carattere pratico che i protagonisti dei sermones oraziani dispensano ai convitati in difficoltà: ma non si sa mai, potrebbero essere utili. Se si è bevuto troppo e si vuole che lo stomaco si rimetta in sesto e recuperi l'appetito iniziale, bisogna mangiare gamberetti arrosto, lumache africane e prosciutto o, ancor meglio, qualche bella salsiccia (Sat. II 4.59 sgg.). Se, poi, non si è ubriachi fradici, ma lo stomaco sazio recalcitra e rifiuta il cibo, un bel bagno caldo è il rimedio migliore per stimolare l'appetito (Epist. I $6.61 \mathrm{sgg}$.). Intestino pigro? Niente paura! Se duro resiste e si rifiuta di accordare una via d'uscita ai cibi che premono, non c'è niente di meglio di un bel piatto di cozze e di vongole, cotte con erbe dalle foglie minute: il tutto accompagnato da qualche bicchiere di vino bianco: di Cos beninteso, altrimenti il successo non è garantito. Se, però, non avete in cantina il vino di Cos, per risolvere i problemi intestinali vi suggerisco, come alternativa oraziana, una scorpacciata di more: però dovete alzarvi presto e coglierle di buon mattino, prima che i raggi del sole diventino troppo violenti, e allora "les jeux sont faits!": il benefico effetto lassativo è garantito (Sat. II 4.21 sgg.). $\mathrm{Ma}$ ora, esaurito il compito di sedicente e improvvisato gastronomo, prima, di amorevole e provvida crocerossina oraziana, poi, non mi resta che ringraziarvi della vostra pazienza. 